\title{
Explosion of a Collapsing Bose-Einstein Condensate
}

\author{
R.A. Duine and H.T.C. Stoof \\ Institute for Theoretical Physics, University of Utrecht, Princetonplein 5, \\ 3584 CC Utrecht, The Netherlands
}

\begin{abstract}
We show that elastic collisions between atoms in a Bose-Einstein condensate with attractive interactions lead to an explosion that ejects a large fraction of the collapsing condensate. We study variationally the dynamics of this explosion and find excellent agreement with recent experiments on magnetically trapped ${ }^{85} \mathrm{Rb}$. We also determine the energy and angular distribution of the ejected atoms during the collapse.
\end{abstract}

PACS number(s): 03.75.Fi, 67.40.-w, 32.80.Pj

Introduction. - Ever since the achievement of BoseEinstein condensation in an atomic gas with attractive interactions [1], it has been an important objective to study in detail the condensate collapse that is predicted to occur [2,3] if the number of condensate atoms exceeds a certain maximum number determined by the strength of the attractive interactions [4]. In the pioneering experiments of Bradley et al. this objective was very difficult to achieve for two reasons. First, the use of doubly spin-polarized atomic ${ }^{7} \mathrm{Li}$ results for these experiments in a maximum number of condensate atoms of only about 1400 atoms, which is too small to allow for nondestructive imaging of the collapse dynamics. Second, the inherent stochastic nature of the growth and collapse cycles that occur because one aims at evaporatively cooling far below the critical temperature [5], prevents sufficient control over the initial conditions of the condensate to perform a sequence of destructive measurements. However, a statistical analysis of the condensate growth and collapse cycles has nevertheless revealed important information on the collapse process [6], and new experimental techniques are presently being applied to overcome in particular the latter of these problems [7].

Complementary to these developments, Cornish et al. have recently been able to overcome both problems in an ingenious experiment with spin-polarized atomic ${ }^{85} \mathrm{Rb}$ [8]. In this experiment one makes use of the fact that the $f=2, m_{f}=-2$ hyperfine ground state of ${ }^{85} \mathrm{Rb}$ has a so-called Feshbach resonance [9] at a magnetic field of about $1.55 \mathrm{mT}$, which offers the opportunity to magnetically tune the interatomic interactions from strongly repulsive to very attractive. As a consequence Cornish et al. were able to first produce a large, stable and essentially pure condensate, and then suddenly switch the interactions from repulsive to attractive to induce a collapse and observe its properties.

Perhaps the most surprising outcome of this experiment is that during the collapse an explosion occurs that ejects a large number of relatively highly energetic atoms from the condensate. A possible mechanism that immediately comes to mind for the production of these energetic atoms are inelastic collisions between condensate atoms that flip a spin and, therefore, convert Zeeman energy into kinetic energy. However, a simple estimate shows that this mechanism generally leads to particles with much too high energies and is also incapable of ejecting so many atoms from the condensate. Similarly it appears that elastic collisions between a condensate atom and an atom in the thermal cloud, which in principle cannot experimentally be excluded to be present, also occur too infrequent to explain the experimental results. We are thus faced with the important theoretical task to determine what physical mechanism is responsible for the observed explosion. It is the main purpose of this Letter to point out that elastic collisions between two condensate atoms can provide such a mechanism.

Elastic Condensate Collisions. - In detail, the scattering process that we have in mind is a collision between two condensate atoms by which one of the atoms is stimulated back into the condensate again but the other atom is ejected out of the condensate. It is important to realize that such a process is forbidden for a homogeneous condensate due to momentum conservation. However, for a trapped gas this is no longer true, because the condensate now occupies a band of low-lying momentum states. Note also that momentum and energy conservation ensure that the ejected atoms will automatically have, as compared to inelastic spin-flip collisions, small kinetic energies and can thus remain trapped as seen in experiment. To see if the above mechanism can also explain the large amount of ejected atoms, we need to calculate the rate associated with this process.

This can be most easily achieved by using the fact that we are dealing with a two-body interaction process. As a result the rate of change of the total number of condensate atoms $N_{\mathrm{c}}(t)$ is related to the instantaneous interaction energy of the condensate $E_{\mathrm{c}}^{\text {int }}(t)$ by

$$
\frac{d N_{\mathrm{c}}(t)}{d t}=\frac{2}{\hbar} \operatorname{Im}\left[E_{\mathrm{c}}^{\mathrm{int}}(t)\right] .
$$

In the Bogoliubov theory of the dilute Bose gas we simply have that

$$
E_{\mathrm{c}}^{\mathrm{int}}(t)=\frac{T^{2 \mathrm{~B}}}{2} \int d \mathbf{x}|\phi(\mathbf{x}, t)|^{4},
$$


which in momentum space reads

$$
\begin{aligned}
& E_{\mathrm{c}}^{\mathrm{int}}(t)=\frac{1}{2}\left(\prod_{i=1}^{4} \int \frac{d \mathbf{k}_{i}}{(2 \pi)^{3}}\right) \phi^{*}\left(\mathbf{k}_{4}, t\right) \phi^{*}\left(\mathbf{k}_{3}, t\right) \\
& \times(2 \pi)^{3} \delta\left(\mathbf{k}_{4}+\mathbf{k}_{3}-\mathbf{k}_{2}-\mathbf{k}_{1}\right) T^{2 \mathrm{~B}} \phi\left(\mathbf{k}_{2}, t\right) \phi\left(\mathbf{k}_{1}, t\right) .
\end{aligned}
$$

Here the two-body $\mathrm{T}$ (ransition) matrix equals $T^{2 \mathrm{~B}}=$ $4 \pi a \hbar^{2} / m$ in terms of the $s$-wave scattering length $a$ and the mass $m$ of the ${ }^{85} \mathrm{Rb}$ atoms, and $\phi(\mathbf{x}, t)$ is the condensate wave function. In this approximation the condensate interaction energy is thus purely real and the number of condensate atoms is conserved.
However, a microscopic derivation [10,11] shows that in Eq. (3) the expression $(2 \pi)^{3} \delta\left(\mathbf{k}_{4}+\mathbf{k}_{3}-\mathbf{k}_{2}-\mathbf{k}_{1}\right) T^{2 \mathrm{~B}}$ should in principle be replaced by the many-body $\mathrm{T}$ matrix element $T^{\mathrm{MB}}\left(\mathbf{k}_{4}, \mathbf{k}_{3}, \mathbf{k}_{2}, \mathbf{k}_{1}\right)$, whose real part is at low temperatures indeed well approximated by the Bogoliubov result $(2 \pi)^{3} \delta\left(\mathbf{k}_{4}+\mathbf{k}_{3}-\mathbf{k}_{2}-\mathbf{k}_{1}\right) T^{2 \mathrm{~B}}$. However, the many-body $\mathrm{T}$ matrix describes also real (incoherent) collisions taking place in the gas and as a result acquires an imaginary part that can be obtained from an optical theorem [10]. Being interested in the ejection of relatively highly energetic particles, we can in first instance neglect the mean-field effects on the corresponding intermediate state to obtain

$$
\begin{aligned}
& \operatorname{Im}\left[T^{\mathrm{MB}}\left(\mathbf{k}_{4}, \mathbf{k}_{3}, \mathbf{k}_{2}, \mathbf{k}_{1}\right)\right] \\
& \quad=-2 \pi\left(T^{2 \mathrm{~B}}\right)^{2} \int \frac{d \mathbf{k}}{(2 \pi)^{3}} \delta\left(\epsilon\left(\mathbf{k}_{2}\right)+\epsilon\left(\mathbf{k}_{1}\right)-\epsilon(\mathbf{k})-\epsilon\left(\mathbf{k}_{2}+\mathbf{k}_{1}-\mathbf{k}\right)\right) \phi\left(\mathbf{k}_{4}+\mathbf{k}_{3}-\mathbf{k}, t\right) \phi^{*}\left(\mathbf{k}_{2}+\mathbf{k}_{1}-\mathbf{k}, t\right)
\end{aligned}
$$

where $\epsilon\left(\mathbf{k}_{i}\right)=\hbar^{2} \mathbf{k}_{i}^{2} / 2 m, \hbar \mathbf{k}$ is the momentum of the ejected atom, and the dependence on the condensate wave function is caused by the effect of Bose enhancement of scattering into already occupied states. Interestingly, the latter is here nondiagonal in momentum space due to the inhomogeneity of the condensate.

Notice that from a field-theoretical point of view, we have in the above manner just arrived at an evaluation of the Feynman diagram drawn in Fig. 1. We thus conclude that we are effectively dealing with a three-body process. Indeed, by taking the functional derivative $\delta / \delta \phi^{*}(\mathbf{x}, t)$ of our result for the condensate interaction energy, we can obtain an imaginary three-body correction term to the Gross-Pitaevskii equation of the condensate, which provides another way of deriving the desired ejection rate. It is also worth mentioning that we qualitatively expect mean-field effects to reduce the above ejection rate in the case of a gas with positive scattering length, because it then costs energy to remove a particle from the condensate. Such an effect, however, does not occur for the negative scattering length case of interest here.

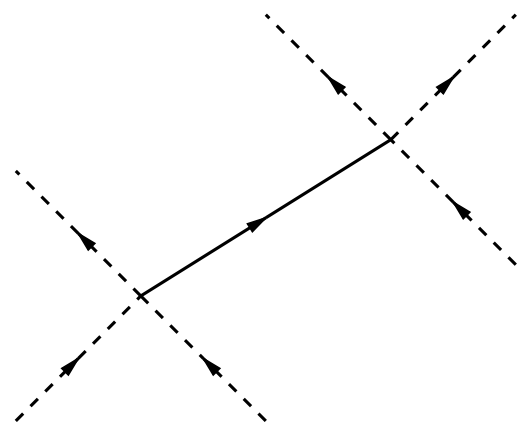

FIG. 1. The imaginary part of this Feynman diagram determines the rate for ejection of atoms out of the condensate due to elastic condensate collisions. The dashed lines correspond to condensate atoms and the full line to a noncondensate atom.
Collapse Dynamics. - To make further progress we need to determine the condensate wave function during the collapse. We do this by solving the Gross-Pitaevskii equation variationally, taking for the wave function an anisotropic Gaussian with time-dependent widths denoted by $q_{x}(t), q_{y}(t)$, and $q_{z}(t)$. In detail we use, apart from a for our purposes irrelevant phase factor,

$$
\begin{aligned}
\phi(\mathbf{x}, t) & =\sqrt{N_{\mathrm{c}}} \prod_{j}\left(\frac{1}{\pi q_{j}^{2}(t)}\right)^{1 / 4} \\
& \times \exp \left\{-\frac{x_{j}^{2}}{2 q_{j}^{2}(t)}\left(1-i \frac{m q_{j}(t)}{\hbar} \frac{d q_{j}(t)}{d t}\right)\right\} .
\end{aligned}
$$

The reason for this Gaussian ansatz is that it is the appropriate description of the condensate after the explosion has occurred and the remnant contains only a relatively small amount of atoms [3, 12]. Moreover, it is well-known that a Gaussian ansatz gives excellent results for the collective modes of the condensate, even when the number of atoms in the condensate is so large that the Gaussian approximation for the condensate wave function is strictly speaking no longer valid [13. We thus expect it to give physically sensible results for the dynamics of the collapse at all times.

Within this approach the explosion of the collapsing condensate is described by the 'classical' equations of motion

$$
m \frac{d^{2} q_{j}(t)}{d t^{2}}=-\frac{\partial}{\partial q_{j}} V\left(\mathbf{q}(t) ; N_{\mathrm{c}}(t)\right)
$$

with a potential energy that is equal to

$$
V\left(\mathbf{q} ; N_{\mathrm{c}}\right)=\sum_{j}\left(\frac{\hbar^{2}}{2 m q_{j}^{2}}+\frac{m \omega_{j}^{2} q_{j}^{2}}{2}\right)+\sqrt{\frac{2}{\pi}} \frac{a \hbar^{2} N_{\mathrm{c}}}{m q_{x} q_{y} q_{z}}
$$


for an anisotropic harmonic trapping potential with spring constants $m \omega_{j}^{2}$. Moreover, these equations are coupled to the rate equation for the number of condensate atoms that follows from Eqs. (11) and (14). In the anisotropic case this cannot be worked out fully analytically and we have to determine the ejection rate numerically. However, in the isotropic case we simply find that

$$
\frac{d N_{\mathrm{c}}(t)}{d t}=-\frac{2 \sqrt{5}}{\pi} \frac{a^{2} \hbar N_{\mathrm{c}}^{3}(t)}{m q^{4}(t)} .
$$

The dependence of the right-hand side on $N_{\mathrm{c}}(t)$ and $q(t)$ is somewhat unusual, because if we had discussed the effect of inelastic two-body processes the rate of change of the number of condensate atoms would be proportional to $N_{\mathrm{c}}^{2}(t) / q^{3}(t)$. The additional factor of $N_{\mathrm{c}}(t)$ is easily understood and reflects the fact that the ejection of the atoms is Bose stimulated. As mentioned previously, our mechanism therefore effectively behaves as a three-body process. The additional factor of $1 / q(t)$ is more subtle and shows that, if the condensate collapses and the wave function becomes more spread out in momentum space, more atoms can satisfy the energy and momentum constraints for the ejection. Having said this, we should make sure that the dominant contribution to the integration in the right-hand side of Eq. (伍) comes from momenta $\hbar \mathbf{k}$ that lie outside the band of momenta occupied by the condensate. We can most conveniently achieve this by including in the integrand the factor $\left(1-|\phi(\mathbf{k}, t)|^{2} /|\phi(\mathbf{0}, t)|^{2}\right)$, which smoothly interpolates between 0 for $\mathbf{k} \cdot \mathbf{q} \ll 1$ and 1 for $\mathbf{k} \cdot \mathbf{q} \gg 1$. In the following we always use this smooth cut-off, because atoms with momenta below the cut-off are most likely stimulated back into the condensate and do not contribute to the ejection rate. It has, however, only a small quantitative effect as can be seen explicitly in the isotropic case, where the right-hand

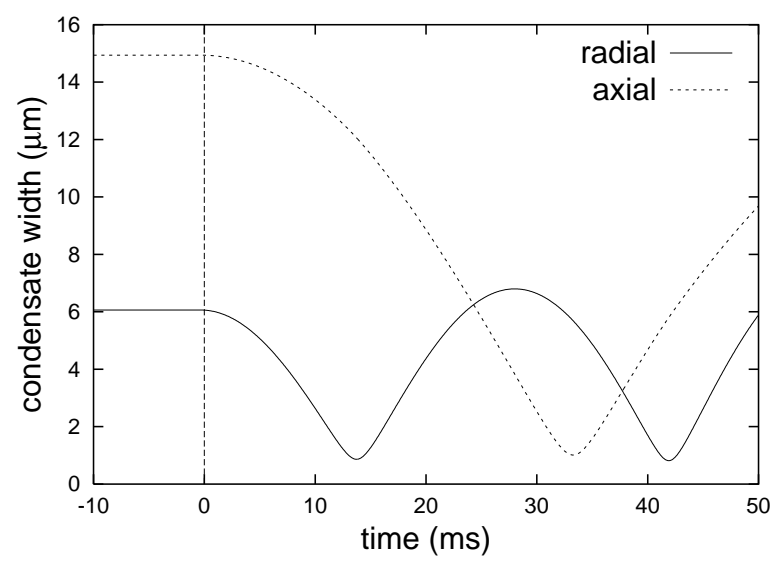

FIG. 2. The radial and axial widths of the condensate during a collapse induced by a sudden change of sign in the scattering length. At the origin of the time axis the scattering length vanishes. See text for more details.

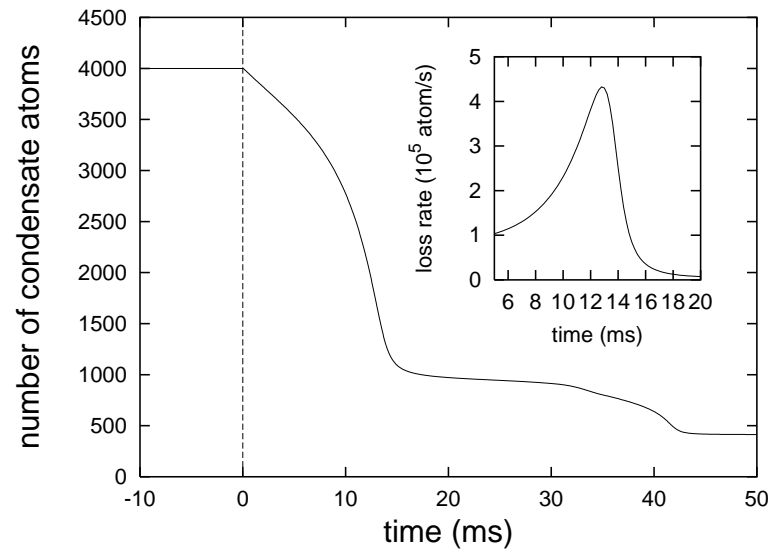

FIG. 3. The number of atoms in the condensate during a collapse induced by a sudden change of sign in the scattering length. The inset shows the ejection rate.

side of Eq. (8) just becomes multiplied by the factor $(1-(8 / 25) \sqrt{2 / 5}) \simeq 0.8$.

We have solved the above set of coupled equations for the conditions of the experiment performed by Cornish et al. [8]. In this experiment one uses a cigar-shaped magnetic trap with a radial frequency of $\omega_{r}=\omega_{x}=\omega_{y}=$ $2 \pi \times 17.5 \mathrm{~Hz}$ and an axial frequency of $\omega_{z}=2 \pi \times 6.8$ Hz. Moreover, one first makes a large condensate with about 4000 atoms and a positive scattering length of 2500 $a_{0}$. The scattering length is then within $0.5 \mathrm{~ms}$ changed to $-60 a_{0}$ by means of a linear ramp in the magnetic bias field. The outcome of our simulation of this experiment is summarized in Figs. 2 and 3, and appears to be in excellent agreement with the preliminary experimental data 14. From these figures we see that the condensate collapses first in the radial direction in approximately $\pi /\left(2 \omega_{r}\right) \simeq 14 \mathrm{~ms}$. During the last part of this collapse an explosion occurs in which about $3 / 4$ of the initial number of atoms is expelled from the condensate. As a result the number of condensate atoms is now less than the maximum number of atoms possible to have a metastable condensate, and the radial collapse of the condensate is turned into a large amplitude oscillation. In principle we can discern at each inner turning point of the radial and axial oscillation an increased loss of condensate atoms, but these are never as dramatic as in the first time, since the condensate is now no longer unstable.

Explosion Process. - To explore the physics of the explosion further, we have also determined the energy and angular distribution of the ejected atoms, which have not yet been examined in detail experimentally. In Fig. A we show the energy distribution at two times during the first radial collapse of the condensate. What is most striking is that at later times the distribution is much broader. This is again caused by the condensate wave function being much more spread out in momentum space at the 


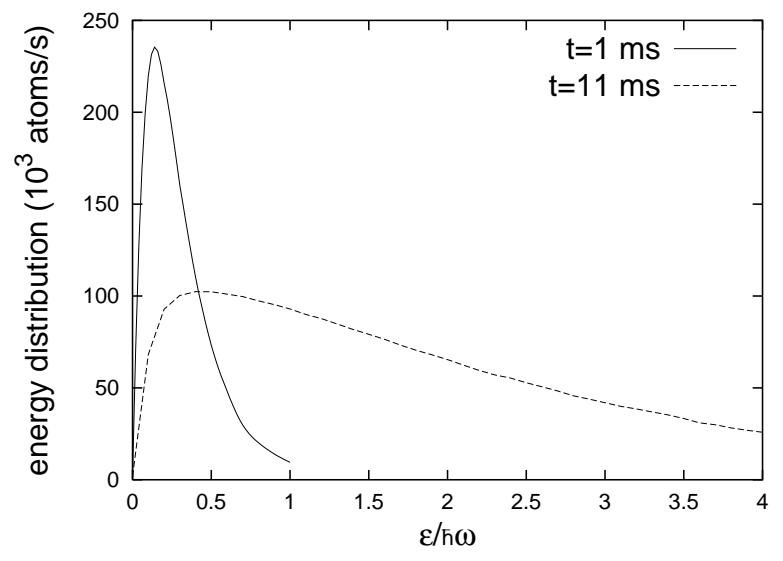

FIG. 4. The energy distribution of the atoms ejected from the condensate at two times during the first radial collapse. We have used a dimensionless energy variable by scaling the energy with $\hbar \omega=\hbar\left(\omega_{r}^{2} \omega_{z}\right)^{1 / 3} \simeq 0.61 \mathrm{nK}$.

later time, so that the ejected atoms can gain much more energy from the condensate collisions. In Fig. 5 we have also depicted at various times the angular probability distribution, which turns out to be essentially independent of the energy of the ejected atoms but is clearly seen to depend strongly on the ratio of the radial and axial widths of the condensate. In particular, at the peak of the explosion the distribution is very anisotropic and almost no atoms are being ejected along the z-axis. This can be understood from the fact that the condensate has at that time a very elongated cigar shape. As a result both the total momentum of the two colliding condensate atoms as well as the momentum of the atom that is stimulated back into the condensate, always have to be directed almost perpendicular to the z-axis. The same is therefore true for the momentum of the ejected atom.

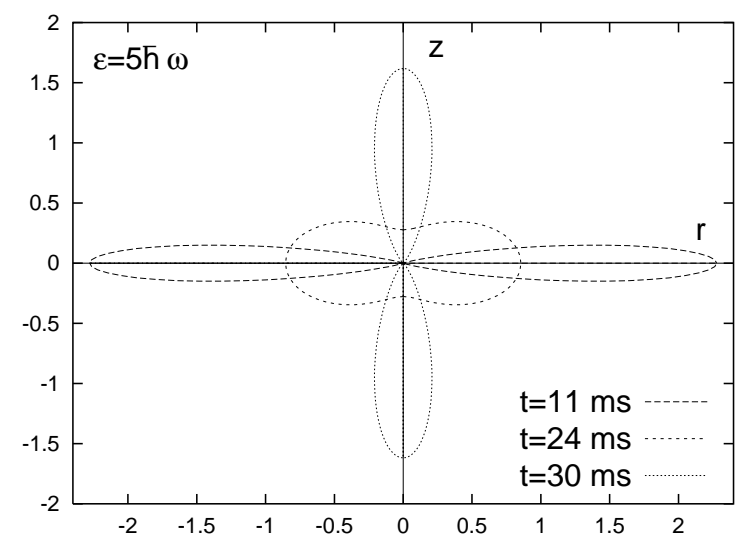

FIG. 5. Polar plot of the angular probability distribution of atoms ejected from the condensate at three different times but at the same energy.

Discussion. - Although we have focused in this Letter on the recent exciting experiments with ${ }^{85} \mathrm{Rb}$, we believe that the new condensate loss mechanism that we have proposed here is also important for the experiments with ${ }^{7} \mathrm{Li}$ and may resolve the existing discrepancy between theory and experiment in that case [6]. In this respect it should be noted that the collapse occurring in the ${ }^{85} \mathrm{Rb}$ experiments is physically quite different and in a sense not so violent than the one occurring in ${ }^{7} \mathrm{Li}$. Roughly speaking the difference is that in the former case the large condensate ejects so many particles that the remnant of the explosion corresponds to a metastable condensate, whereas in the latter case it is precisely this metastable condensate that collapses to even smaller sizes. Possibly another important difference is that in the experiments of Cornish et al. no thermal component is visible, whereas the experiments of Sackett et al. are close to the critical temperature and, therefore, a large thermal cloud is constantly feeding the condensate. We intend to come back to a detailed theoretical treatment of these interesting issues in a future publication.

It is our pleasure to thank Simon Cornish and Carl Wieman for providing us with their experimental data before publication and for stimulating discussions that have initiated the above. This work is supported by the Stichting Fundamenteel Onderzoek der Materie (FOM), which is financially supported by the Nederlandse Organisatie voor Wetenschappelijk Onderzoek (NWO).

[1] C.C. Bradley, C.A. Sackett, J.J. Tollett, and R.G. Hulet, Phys. Rev. Lett. 75, 1687 (1995); C.C. Bradley, C.A. Sackett, and R.G. Hulet, Phys. Rev. Lett. 78, 985 (1997).

[2] E.V. Shuryak, Phys. Rev. A 54, 3151 (1996).

[3] H.T.C. Stoof, J. Stat. Phys. 87, 1353 (1997).

[4] P.A. Ruprecht, M.J. Holland, K. Burnett, and M. Edwards, Phys. Rev. A 51, 4704 (1995); M. Houbiers and H.T.C. Stoof, Phys. Rev. A 54, 5055 (1996); T. Bergeman, Phys. Rev. A 55, 3658 (1997).

[5] C.A. Sackett, H.T.C. Stoof, and R.G. Hulet, Phys. Rev. Lett. 80, 2031 (1998).

[6] C.A. Sackett, J.M. Gerton, M. Welling, R.G. Hulet, Phys. Rev. Lett. 82, 876 (1999).

[7] R.G. Hulet, private communication.

[8] S.L. Cornish, N.R. Claussen, J.L. Roberts, E.A. Cornell, C.E. Wieman (unpublished, cond-mat/0004290).

[9] W.C. Stwalley, Phys. Rev. Lett. 37, 1628 (1976); E. Tiesinga, B.J. Verhaar, and H.T.C. Stoof, Phys. Rev. A 47, 4114 (1993).

[10] H.T.C. Stoof, Phys. Rev. A 45, 8398 (1992); J. Low Temp. Phys. 114, 11 (1999).

[11] H. Shi, and A. Griffin, Phys. Rep. 304, 1 (1998).

[12] M. Ueda and A.J. Leggett, Phys. Rev. Lett. 80, 1576 (1998).

[13] V.M. Perez-Garcia, H. Michinel, J.I. Cirac, M. Lewenstein, and P. Zoller, Phys. Rev. Lett. 77, 5320 (1996).

[14] S.L. Cornish and C.E. Wieman, private communication. 